\title{
Fourier Methods in Imaging
}

Roger L. Easton, Jr., 930 pp., ISBN 978-0-470-68983-7, John Wiley and Sons (2010), \$165 hardcover.

Reviewed by Paul J. Kane, Kodak Research Laboratories, Rochester, New York.

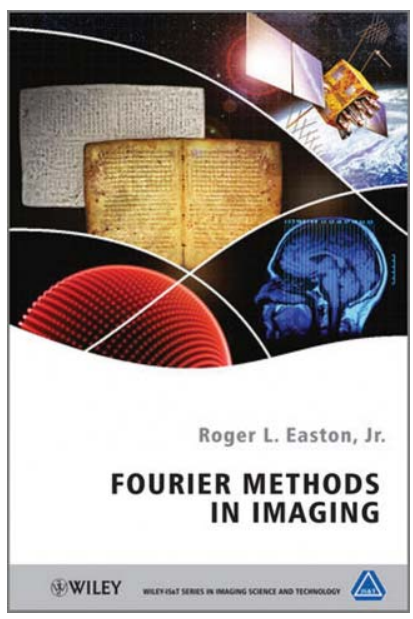

Fourier analysis is a welldeveloped branch of mathematics with wide-ranging applications in statistics, the physical sciences, engineering, and economics. For many readers of this journal, the use of Fourier analysis is as natural as algebra or trigonometry. Over the years, many textbooks have been written on the use of the Fourier transform in optics and imaging by well-known authors. One may ask, therefore, why this book?

The answer is that this book provides the imaging community with a single reference on the subject of Fourier methods that uses consistent notation, includes a wealth of supporting material, and develops the subject within a consistent framework. The author is a long-time faculty member at Rochester Institute of Technology's Center for Imaging Science, where he is an awardwinning teacher and researcher. This text is the result of many years of teaching and research experience, and the results are impressive.

Chapter 1 introduces the reader to the concepts of the "imaging chain," and what are termed the three basic tasks of imaging: 1. prediction of the image function, given the object function; 2. inference of the object function, given the image function; and 3. system analysis, or inference of the system function, given both the object and image functions. The chapter concludes by briefly discussing several types of imaging modalities, namely optical, radiographic, and gamma ray, and provides specific examples of applications of Fourier methods to imaging problems, such as the diagnosis of the Hubble Space Telescope aberrations, and computed tomographic radiography.

Chapters 2 through 5 of the book provide a compact review of areas of mathematics needed to understand Fourier analysis in both the continuous and discrete (spatially or temporally sampled) domains. This begins with basic concepts such as linearity and shift invariance, differences between discrete and continuous functions, and symmetry properties of functions. The discussion quickly moves through linear algebra, complex variables, and eigenfunctions of continuous operators. The author not only provides basic principles and key results in these areas, but also gives some advanced material that is needed for later chapters. This serves as a refresher for readers already exposed to the material, as well as a primer on the author's notation. Less experienced readers may find these sections a bit dense; the material here is often spread out over entire textbooks. Also, there are somewhat fewer graphs and figures here, as compared to the rest of the book.

Chapters 6 and 7 introduce the 1D and 2D "special functions" that are commonly used in Fourier optics and imaging, such as the rect, triangle, sinc, and Dirac delta functions. The pace of the text slows a bit at this point, with graphs and images used frequently. The author has included much material on the use of the Dirac delta and comb (or sampling) functions, including some of the trickier aspects of these, such as raising the delta to a power, derivatives of the delta, and the delta with a functional argument. Also discussed here are the application of Fourier methods to the analysis of random variables and stochastic functions.

Chapters 8 through 11 provide the fundamentals of linear systems theory, then introduce 1D and multidimensional Fourier transforms. In particular, Chapter 8 defines and describes linear, shift-invariant, and LSI operators in some detail, then proceeds to convolution and correlation, including the definition of autocorrelation and autocovariance for stochastic functions. Chapter 9 introduces the 1D Fourier transform, beginning with a general discussion of integral transforms, integral inner products, and projections. This leads to a consideration of even and odd sinusoids as the reference function in such integrals, and ultimately to the introduction of the Hartley transform. Next, the complex exponential reference function is introduced, which naturally leads to the Fourier transform. The chapter then covers all of the relevant Fourier transform theorems, the Fourier transforms of the 1D special functions, and also the effects of selected nonlinear operations on the transform. Many graphical examples are provided here. Chapter 10, relatively short by comparison, extends the analysis to multidimensional transforms, although the discussion is mostly directed towards $2 \mathrm{D}$ functions. Issues specific to two dimensions are addressed, such as separable functions and the mathematical results of rotation. The 1D graphs of Chapter 9 give way to numerous $2 \mathrm{D}$ grayscale images that illustrate the theorems. The case of $2 \mathrm{D}$ functions with circular symmetry is dealt with separately in Chapter 11, which covers the Hankel transform and its attendant theorems.

Chapter 12 is devoted to the Radon transform, which is of great significance in medical imaging applications. The treatment is more extensive than I have found in any other book of this kind. It includes discussion of the projection slice (or central slice) theorem, and many examples of the Radon and central-slice transforms of special functions. I found that the short final section of this chapter comparing the Fourier and Radon transforms of images left me wanting to see more examples. However, the extended treatment here ought to inspire readers less familiar with the Radon transform to think about new applications for this analysis.

Chapter 13 covers two methods of approximating Fourier transforms, using the moment theorem and the method of stationary phase. These are valuable both in terms of numerical analysis, as well as in gaining insight into transforms that do not have a straightforward analytical solution. Also, the Central Limit 
Theorem, width metrics in the spatial and frequency domains, and the Fourier uncertainty relation are discussed.

Chapters 14 through 15 provide a thorough discussion of sampling, aliasing, and quantization effects, and the discrete Fourier transform (DFT), with an introduction to the discrete cosine transform that forms the basis for image compression standards. This includes the Whittaker-Shannon sampling theorem, interpolation in the space (or time) and frequency domain, and an exploration of the fast Fourier transform (FFT) algorithm. The practical details of DFT/FFT use are explored, which is valuable material for users of canned FFT algorithms, which includes just about everyone.

Chapters 16 through 18 comprise an extensive description of filtering. In particular, Chapter 16 covers magnitude filtering in both one and two dimensions. The various types of filtering operations are described in detail, including lowpass, highpass, bandpass, and bandstop (also called band-reject) filtering. All of these are illustrated with examples and graphics. The chapter ends with a very brief introduction to wavelets, illustrating the roots of wavelet decomposition in linear filtering theory. Chapter 17 describes phase or allpass filters, including constant, linear, and quadratic filters. The latter are of great interest in optics and radar. Also included are higher order phase filters and random phase filters, which are important in analysis of turbid media. The chapter concludes with the optical correlator and imaging of phase-only objects. Chapter 18 investigates the general case of magnitude-phase filters, with examples of simple operations such as differentiation and integration. It also includes a discussion of causality and the simple harmonic oscillator, which sheds light on some of the more subtle features of linear systems theory.

Chapter 19 covers well-known applications of linear filters, the first being deconvolution and inverse filtering. Analysis of common imaging artifacts such as blur from a moving or rotating camera (uniform averaging) and noise is presented, leading into discussion of optimum estimators, the Wiener filter, and matched filtering. The discussion in this chapter follows primarily 1D examples tied to analysis of 1D continuous functions. With that background, Chapter 20 discusses linear filtering in discrete (sampled) systems, which is more directly related to image processing. Included in the discussion are averaging (lowpass) and differencing (highpass) filters, the Laplacian and sharpening operators, and approximate discrete reciprocal filters.
Chapters 21 and 22 are devoted to what would typically be called physical optics, the former devoted to imaging in monochromatic light (coherent optical systems) and the latter devoted to incoherent optical systems. Given the perspective of this text, this leads naturally to a consideration of the relevant mathematical forms: Fresnel diffraction is described by the Fresnel transform, and Franhoufer diffraction is described by the Fourier transform. Along with the emphasis on application of Fourier analysis and transforms, a good discussion of the physics is included. There is good material here on the ray and wave optics models, along with detailed descriptions of the interaction of waves with phase objects, lenses, and knife edges. In the latter chapter, there are discussions of coherence, extensions to polychromatic light, and of course the point-spread function and modulation transfer function of incoherent optical systems.

The book concludes with a chapter on holography, including computer-generated holography, matched filtering, and synthetic aperture radar.

While the list of references is good, I would have liked to see more references embedded in the text in appropriate places. Perhaps this could be considered for the second edition, which I am confident will be produced. Overall, this is an excellent text, appropriate for the graduate student approaching this material for the first time, and for the seasoned professional looking for an up-to-date reference.

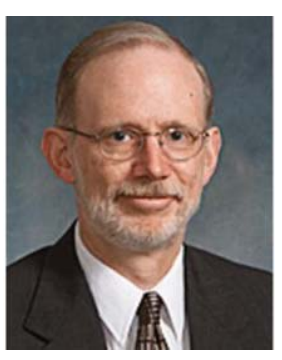

Paul Kane is a senior principal scientist with Kodak Research Labs in Rochester, New York. He received his MS in optics from the University of Rochester and has been with Kodak since 1986. His work has included imaging system modeling, metrology, and quality metrics for analog and digital imaging systems. His recent work has been in the development of OLED and laser projection displays, and 3D imaging systems. He holds 12 U.S. Patents, and he received the Kodak Research Laboratory's C.E.K. Mees Award for work on pixel patterns and image-processing algorithms for OLED displays. He is a member of SPIE and OSA. 